John Carroll University

Carroll Collected

Theology \& Religious Studies

$3-2006$

\title{
Response to Richard B. Miller's Children, Ethics, and Modern Medicine
}

Paul Lauritzen

John Carroll University, plauritzen@jcu.edu

Follow this and additional works at: http:/ / collected.jcu.edu/theo_rels-facpub

Part of the Ethics in Religion Commons

\section{Recommended Citation}

Lauritzen, Paul, "Response to Richard B. Miller's Children, Ethics, and Modern Medicine" (2006). Theology \& Religious Studies. 65. http://collected.jcu.edu/theo_rels-facpub/65

This Article is brought to you for free and open access by Carroll Collected. It has been accepted for inclusion in Theology \& Religious Studies by an authorized administrator of Carroll Collected. For more information, please contact connell@jcu.edu. 


\title{
RESPONSE TO RICHARD B. MILLER'S \\ CHILDREN, ETHICS, AND MODERN $M E D I C I N E$
}

\author{
Paul Lauritzen \\ Department of Religious Studies \\ John Carroll University
}

Although there is much in this volume that would repay careful study, I want in this brief essay to focus on what I take to be the theoretical heart of the book, namely, Chapter 5, on basic interests. It is in this chapter that Miller takes up the important task of defending liberalism as a political theory that can generate an account of pediatric ethics that is compatible with the religious and moral pluralism of our society. My plan is briefly to set out Miller's claim that liberal theory can define both what is permissible and what is impermissible in the medical treatment of children without endorsing any particular comprehensive theory of the good. I am deeply sympathetic to his effort to provide a normative account of pediatric ethics along liberal lines (Miller 2003, 130), but I want to explore the contours of this normative understanding by focusing on a couple of case studies that may lead us either to question the contrast Miller draws between basic interests and best interests or to call for a more expansive understanding of basic interests or both.

In order to understand what Miller is up to in his chapter on basic interests, it is important to see the dilemma posed for a political liberal wrestling with issues of pediatric ethics. The basic problem is that in caring for many children and adolescents it will not be possible to rely on a child's autonomous choice to secure well-being. Caring for pediatric patients requires promoting beneficence rather than autonomy, and this in turn requires defining what is in the patient's interest, often in the absence of any meaningful measure of how the patient him- or herself would define this interest. This creates what Miller refers to as "the problem of moral pluralism." "Without a socially shared vision of what it means to flourish as a human being," Miller writes, "it is impossible to determine how to protect a patient's interest. What is 'good' for a person is up for grabs in a culture that lacks a shared understanding of the ends of human life, for without the idea of such ends we cannot determine how to promote that person's interest" (Miller 2003, 120). In the absence of any shared vision of the good and without any sense of the patient's own vision of the good, we seem to be without a moral compass.

I cannot do full justice to Miller's carefully developed and subtle response to this problem, but the gist of his solution is that we must distinguish liberalism as a political theory from liberalism as a comprehensive theory. Liberalism as a political theory is able to maintain neutrality regarding comprehensive visions of the good while nevertheless identifying primary goods that we have a duty to provide to those in need. In developing this response, Miller draws on Rawls's idea of an "overlapping consensus" of comprehensive theories of the good, a consensus that supports at least a "thin" theory of the good. Distinguishing between thick and thin theories of the good allows us to draw a related distinction between basic interests and best interests. Basic interests are concerned with the primary goods that everyone needs no matter what his or her comprehensive vision is; best interests necessarily require a more full-bodied account of the good. 
With these distinctions in mind, Miller's response to the problem of pluralism in pediatric medicine is this: When we cannot know a child's or an adolescent's comprehensive vision of the good, we have a duty to promote her basic interests, not her best interests. That is, we have a duty to provide the primary goods that children need and whatever else they may want, now or in the future. In the case of children, that means "coming to terms with the goods of physical, intellectual, and emotional welfare; respect; and the right to an open future" (Miller 2003, 126).

I confess that I find this account of political liberalism and its normative commitments enormously appealing. I especially find it so in a society where some groups appear increasingly willing to seek to impose their comprehensive visions of the good on others. Nevertheless, I wonder whether, in the end, Miller's account of basic interests is substantial enough to secure the well-being of children in a society that, for all its talk of the importance of children, does not really value children.

To give a sense of my concern, I want to begin with a case that is only marginally related to pediatric ethics; it does, however, highlight the difficulty of securing the well-being of children in our society. The case involves what I sometimes think of as the "silent" sex abuse crisis in the country. This crisis is not the much-discussed sex abuse scandal in the Catholic Church, but the almost daily focus on the sexual activities of children on the daytime TV talk shows. ${ }^{1}$ Alas, with ever growing frequency, the daily talk shows exploit children, especially teenage girls, by encouraging them to recount the salacious details of the sex lives for a studio and TV audience. Jenny Jones, Maury Povich, Sally Jesse Raphael, Montel Williams, Jerry Springer-all have aired such shows.

For anyone with even passing acquaintance with these shows, it will come as no surprise that Jenny, or Jerry will do almost anything to improve ratings. (If you are not familiar with these shows, the title of a segment from a Jenny Jones show-no doubt run during "sweeps" week-will give you a sense of this fare: "Somebody Give My Daughter A Whipping, She Wants to Make Her Money Stripping.") Now it is perhaps unfair to Miller to suggest that his account of securing children's basic interests may require him to shout down Jerry Springer, and I certainly do not want symbolically to break a chair over Miller's head, as one of Jerry's guest might literally do, but there is a serious issue to be considered here: Can Miller's account explain the harm that is done to these teenagers in a way that generates a duty to prevent such exploitation?

In responding to this question, it is important to note that it would be a mistake to dismiss such shows as marginal and beneath contempt, for it is not just Jenny and Jerry who exploit teenagers in this way. Oprah and Dr. Phil do so as well; and whatever you think of these shows, there is no disputing that they have a huge mainstream following. Yet these two "champions" of mental health in this country clearly - at least to me it is clear-exploit children. Consider, for example, a segment on the Oprah show from when Dr. Philwas still a regular on the show. ${ }^{2}$ Entitled, "Dr. Phil on Alarming Sexual Behavior," the segment consisted of interviews with three girls, fourteen and fifteen years old, who appeared on the show with their mothers. The girls described the way in which they had casually provided boys with oral sex, sometimes in the school bathroom, sometimes on the bus to or from school. We learn that one of the girls had begun doing this when she was thirteen years old. The program was punctuated by prerecorded interviews with other girls and boys who described their various sexual activities and partners.

What should we make of the fact that Dr. Phil interviews teenagers on national television about their sexual practices? Dr. Phil will, of course, say that he is just "getting real," that 
teenage sexuality is a problem that will not go away by ignoring it. As a parent of teenagers, I certainly would agree with him about that. Yet, putting teenagers on national television to talk about their sex lives is arguably to exploit them. It violates their privacy and exposes them to extraordinary scrutiny at a particularly vulnerable time in their lives. But does it violate their basic interests? As a political liberal, am I required to ignore the decision of these children's parents who, not sharing my vision of the comprehensive good that would lead them to repudiate such voyeurism, give consent? I do not doubt that Miller would condemn this exploitation of children, but can he do so given his thin theory of the good and the duty to secure only basic interests? In short, is tolerating this sort of instrumental use of children and adolescents the price we pay for the liberal solution to the problem of pluralism? ${ }^{3}$

The second set of cases on which I want to focus intersects more directly with Miller's work, because the cases involve both medical treatment of, and medical research on, children. Specifically, I want to pose for Miller's consideration the use of synthetic growth hormone (GH) for nonhormone deficient children. ${ }^{4}$ As I understand it, recombinant GH is approved for use in children for only three conditions: GH deficiency, Turner's syndrome, and chronic renal disease. Nevertheless, because biosynthetic GH is now readily available and because there is some evidence that GH may increase adult height if administered to children who are short but not suffering from any of the problems for which it is an approved treatment, parents have been requesting $\mathrm{GH}$ treatment for their children and pediatricians have been providing it. Indeed, one study indicated that $94 \%$ of pediatric endocrinologists surveyed would recommend GH therapy for children with so-called idiopathic short stature (Leschek et al., 3140).

I would be interested in Miller's reflections on the therapeutic alliance that permits the general use of GH for children whose only "medical" problem is that they are short. But I want to sharpen the case by focusing on one clinical trial involving GH that raises serious issues about whether a duty to promote children's basic interests is enough. Although I would not go so far as to call the study a "sanitized form of barbarism," to borrow Paul Ramsey's language about pediatric research, in my view it is a study that should not have been conducted. Because Miller is such an insightful observer of pediatric medicine and because I think this study poses an intriguing case for his theory, I am interested to hear what he would say about it.

The study was jointly funded by the National Institute of Child Health and Human Development and the Eli Lilly and Company Growth Hormone Collaborative Group, and it was occasioned by the situation I described above. That is, thousands of children of short stature are receiving $\mathrm{GH}$ in this country despite the fact that there is no definitive evidence that $\mathrm{GH}$ increases adult height and despite the fact that there are risks associated with receiving GH.With that in mind, the authors of this study designed a randomized, double-blind, placebo-controlled trial to study the effect of $\mathrm{GH}$ on adult height in peripubertal children.

Although the results of the study were only recently published (Leschek et al.), the study itself had been in the works for many years and was controversial from the start (see Prentice et al. 1989; Tauer 1994; White 1993). The original study design called for recruiting 80 children ranging in age from 9 to 15 who were not GH deficient but of short stature. The study was to involve an initial four-day evaluation period at National Institutes of Health (NIH), during which subjects would undergo routine blood and urine tests, X-rays, MRIs (magnetic resonance imagings), and behavioral and psychological tests. Each subject was to be photographed nude. Subjects were to return to NIH at six-month intervals for similar workups. For the study itself, subjects were to be randomized to either the HG arm or the placebo arm of the study and both groups would receive subcutaneous injections 3 times per week over the course of study, in some 
cases for up to seven years. Although the precise number of injections could not be determined in advance, the investigators estimated that subjects would receive between 600 and 1,100 injections.

I want shortly to look at how the Institutional Review Board (IRB) and the special review panel at NIH came to approve this study, but before doing so, let me say that I agree with Miller that "the first line of moral responsibility falls on investigators and IRBs... not on parents or guardians who may agree to enroll their children" (Miller 2003, 246). Indeed, I think Miller shows convincingly that Paul Ramsey got the ethics of pediatric research badly wrong by focusing on proxy consent instead of on patient benefit. Focusing on the issue of consent, Ramsey concluded that children may never be subjects of nontherapeutic research because they are not able to consent to such research and "no parent is morally competent" to consent on their behalf. However, as Miller points out, the fact that Ramsey allowed parents to consent to therapeutic research demonstrates that the real issue here is not consent but patient benefit. As Miller puts it: "Had Ramsey seen matters more clearly, he would have argued that canons of loyalty to a child in research settings must begin not by focusing on criteria for validating parental consent, but with determining whether the proposed intervention is in that child's basic medical interest" (Miller 2003, 245). I agree with Miller about this.

With this standard in mind, then, we can return to the GH study. We do not know how the original IRB that approved this study reasoned to its conclusion, but we know that their conclusion was controversial. In fact, in response to criticism from the Foundation for Economic Trends and the Physicians Committee for Responsible Medicine, NIH convened a committee to review the social and ethical issues raised by the GH study. The Review Committee recommended that the short stature study, as they called it, be allowed to go forward (National Institutes of Health 1992). Because the Review Committee published its deliberations in a report, we have an interesting (and unusual) record of moral analysis in this case that deserves attention. To understand the conclusions reached by the Review Committee, it is necessary to review the Department of Health and Human Services (DHHS) guidelines for research involving experimentation on children. (Miller takes these up in chap. 11) According to the relevant guidelines (45 CFR 46, sec. 46.404-46.406), studies involving experimentation on children may be undertaken if the research (a) poses only minimal risk (46.404), (b) involves greater than minimal risk but presents the prospect of direct benefit to the individual subject (46.405), or (c) involves greater than minimal risk, provides no prospect of direct benefit to the individual subject, but is likely to yield generalizable knowledge about the subjects' disorder or condition (46.406). The Review Committee offered an assessment of the GH study under all three sections, and their analysis is instructive.

The first issue was whether the short stature study was acceptable because it represented only minimal risk to the subjects. Here federal guidelines are explicit: "Minimal risk means that the probability and magnitude of harm or discomfort anticipated in the research are not greater in and of themselves than those ordinarily encountered in daily life or during the performance of routine physical or psychological examinations or tests" (46.102). The Review Committee unanimously concluded that the risks to the subjects were more than minimal. The mere fact that children enrolled in the study would receive three shots per week, in some cases for up to seven years, was enough to place the study beyond the threshold of minimal risk. Moreover, the study could increase self-consciousness about height or reinforce a harmful and misguided view that the subjects are "abnormal." As the Review Committee wrote, even though it is "difficult to 
weigh or to calculate the probability of these risks," they are greater than minimal, as defined by the regulations.

The Review Committee then took up the question of whether the study was acceptable under 46.405, that is, research involving greater than minimal risk "but presenting the prospect of direct benefit to the individual subjects." Once again, federal regulations are explicit, even if hard to apply. ${ }^{\mathbf{5}}$ IRBs may approve research involving greater than minimal risk but promising direct benefit under three conditions: (a) the risk is justified by the anticipated benefit to the subjects, (b) the relation of the anticipated benefit to the risk is at least as favorable to subjects as that presented by available alternative approaches, and (c) adequate provisions are made for soliciting the assent of the children and permission of their parents or guardians. In applying 46.405, the Review Committee noted two major difficulties. First, the regulations do not spell out how to determine whether there is a prospect of direct benefit to each individual subject. Second, balancing benefits against risks in this case is a complex undertaking.

Consider the problem of determining whether there is a likelihood of direct benefit to each individual in the study. As the Review Committee points out, one obvious question is whether benefit should be calculated before or after randomization takes place. If the calculation takes place before randomization, then assuming that $\mathrm{GH}$ may be beneficial, each subject in the protocol has a $50 \%$ chance of benefiting. By contrast, if the calculation takes place after randomization, then the subjects in the placebo arm of the study can expect no benefit, given that they will not receive the GH. ${ }^{6}$

According to the Committee, the question of whether the short stature study meets the requirements that either the risk is justified by the anticipated benefit to the subject (46.405(a)) or that the risk/benefit ratio is at least as good as the available alternative approaches (46.405(b)) is extremely difficult to answer in light of "the paucity of good data about the probability of risks, and by the absence of common measures of their significance." Indeed, a minority of the panel believed that the risks were justified by the anticipated benefits; another minority believed that the risks outweighed the benefits; and a third group believed that the calculations were either too speculative or too evenly divided to reach a definitive conclusion. Nevertheless, the Committee was able to reach consensus because they interpreted 46.405 (a) to be a requirement that the study be in equipoise.

The claim of equipoise is, of course, central to any defense of randomized clinical trials (Chard and Lilford 1998; Freedman 1987; Gifford 1995; Karlawish and Lantos 1997). As it is typically understood, equipoise refers to the fact that there is a genuine null hypothesis being tested in the study. Because physicians are committed to providing patients with the best possible treatment, for a randomized clinical trial to be ethically justified there must be uncertainty about whether a new therapy will be at least as effective as the standard treatment.

When there is no proven treatment, there must be uncertainty that the new therapy will not be better than no treatment. The Review Committee clearly invoked this traditional understanding of equipoise. "Clinical equipoise," they wrote, "exists if, after considering the evidence regarding the tradeoff and risks for each treatment strategy, there remains sufficient collective uncertainty regarding which strategy is superior so that opinion would be divided among reasonable medical experts." In the case of the short stature study, clinical equipoise would require that there be genuine medical uncertainty whether providing $\mathrm{GH}$ to nonhormonedeficient children is preferable, given the expected risks and benefits, to not treating at all. And indeed, it was precisely this uncertainty that led to the initiation of the trial in the first place. 
The problem is that by asking primarily about benefit, the framework of equipoise is likely to obscure the possibility of real harm. So long as there is no proven treatment, evaluating a traditional placebo-controlled study involves weighing two somewhat uncertain probabilities of benefit. Asking about equipoise thus involves comparing the study drug to the placebo and asking whether there is a roughly equal likelihood of benefit from each. So the comparison is between taking the study drug and taking the placebo. But what about cases, like the short stature study, where the administration of the placebo itself may cause harm? In such cases, the fact that the likelihood of benefit is roughly equal between a treatment arm of the study and the placebo arm cannot be morally determinative.

Where placebos may harm, the choice is not merely between taking the study drug and taking a placebo. There is a third choice: doing nothing. While not participating in the study is, of course, always an option with a traditional placebo-controlled trial, not participating and taking a placebo are essentially the same in the standard drug study, at least with respect to harm. And this is decidedly not the case in studies where placebos may harm. Although the possibility of benefit may be roughly equivalent when the two arms are compared, and although participating in the study may provide greater likelihood of improvement than doing nothing, participating in such a study will expose a subject to harm that he or she would not be exposed to outside of the study.

My conclusion is thus that the short stature study should not have been conducted. I am inclined to think that Miller would agree. For example, at one point, he writes: "The rationale for allowing nontherapeutic research that includes a minor increase over minimal risk cannot be justified. According to the federal regulations, studies may impose a 'minor increase over minimal risk' when the research is likely to yield generalizable knowledge about the subject's disorder which is of vital importance for understanding or ameliorating that disorder.

This permission allows adults to enroll children if the costs to the research subject are outweighed by the potential benefits to society and medicine." But, says Miller, "Justifying pediatric research in this way instrumentalizes children. It allows the ends to justify the means and increases risks to a vulnerable population" (Miller 2003,

253-254).

At first blush, this statement would seem to rule out the GH study because the placebo arm of the study arguably involves nontherapeutic research that includes more than minimal risk for the sake of possibly benefiting society and medicine. The problem here is twofold. First, calculating risks and benefits before randomization leads to the conclusion that the study is potentially beneficial and therapeutic, for all the children enrolled. If so, Miller's comments here cannot be interpreted as weighing against this study. Second, and more important, I am not sure that Miller's account helps us to see that the basic problem with the GH study is that it presupposes that being short is a medical disorder. Commenting on the federal guidelines that allow for a proportionate balance of risk and benefit in research that involves greater than minimal risk, Miller writes: “. . . harm to individuals is justified in medical settings on the condition that it is offset by the pursuit of patient benefit. I see no difficulty with this idea as it applies to research with sick children" (Miller 2003, 251). I agree with this statement; I just do not think the children in the GH study are "sick." They are not hormone deficient, they do not have Turner's syndrome, and they are not suffering from chronic renal insufficiency. They are simply short. ${ }^{7}$

As I indicated above, Miller does an admirable job showing that the concepts of "minimal risk" and "minor increase over minimal risk" are "subject to widely different 
interpretations by IRBs" and I endorse his call for a national commission that would, among other things, seek to clarify these standards. But I wonder if the problem is not broader and deeper than this. I wonder whether, in the absence of a comprehensive theory of the good, or at least a thicker account of the good than Miller provides, we are able even to recognize how bizarre it is to refer to being short as an "idiopathic" disorder. Whether or not Miller agrees with me about the dangers of a thin theory of the good here, my hope is that this excellent volume will lead to a sustained national conversation about pediatric ethics. As Miller skillfully demonstrates, such a conversation is badly needed. Fortunately, with this volume we have a point of departure (and an outline) for an extraordinarily fruitful conversation.

\section{NOTES}

1) I am indebted to Mark Sheldon at Northwestern University for bringing this issue to my attention and for help in formulating the moral issues at stake here.

2) I wish I could report that Dr. Phil has moved away from this sort of voyeuristic practice now that he has his own show, but a search of his web site suggests otherwise. A search for the keyword "teen" returns 65 entries, including, among others, one for "Sex Talk: Shocking Attitudes about Sex" and "Oral Sex from Teens Across America."

3) One response that is open to Miller, of course, is to suggest that any practice that exploits adolescent sexuality in this way will be ruled out by an overlapping consensus that recognizes that fostering self-esteem in teenagers is necessary to healthy adult civic engagement and thus is necessary for any society to flourish, no matter its conception of the comprehensive good. The problem with this move is that our society seems not the least bit concerned about the exploitation of adolescent sexuality in the way that this kind of response seems to require.

4) The discussion of the growth hormone study that follows was originally written in collaboration with Martin Smith and is deeply indebted to his thoughtful analysis of this case.

5) Miller does an excellent job explaining how these standards appear to be but are not clear.

6) One line of argument would be to suggest that subjects in the placebo arm stand to benefit from a placebo effect and so, even apart from the kind of indirect benefits discussed by the Review Committee, the placebo group can expect to receive direct benefit. The Review Committee explicitly rejected this line of argument (National Institutes of Health 1992, 12).

7) I do not mean to dismiss the difficulties that result from being short, some of which may involve considerable suffering. My point is that I do not think that shortness should be defined as a "sickness."

\section{REFERENCES}

Chard, J. A., and R. J. Lilford

1998 "The Use of Equipoise in Clinical Trials." Social Science Medicine 47.7:891-8.

Freedman, Benjamin

1987 "Equipoise and the Ethics of Clinical Research." The New England Journal of Medicine 317:141-5.

Freedman, Benjamin, Kathleen Cranley Glass, and Charles Weijer 
1996 "Placebo Orthodoxy in Clinical Research II: Ethical, Legal and Regulatory Myths." Journal of Law, Medicine, and Ethics 24:252-9.

Gifford, Fred

1995 "Community Equipoise and the Ethics of Randomized Clinical Trial." Bioethics 9.2:127-48.

Karlawish, Jason H. T., and John Lantos

1997 "Community Equipoise and the Architecture of Clinical Research." Cambridge Quarterly of Healthcare Ethics 6:385-96.

Leschek, E. W., S. F. Rose, and J. A. Yanovski, et al.

2004 "Effect of Growth Hormone Treatment on Adult Height in Peripubertal Children with Idiopathic Short Stature: A Randomized, Double-Blind, Placebo-Controlled Trial." The Journal of Clinical Endocrinology and Metabolism 89.7:3140-8.

Miller, Richard B.

2003 Children, Ethics, and Modern Medicine. Bloomington, Ind.: Indiana University Press. National Institutes of Health

1992 Report of the NIH Growth Hormone Protocol Review Committee. October 2.

Prentice, Ernest D., Dean L. Antonson, Andrew Jameton, Benjamin Graber, and Thomas Sears

1989 "Can Children Be Enrolled in a Placebo-Controlled Randomized Clinical trial of Synthetic Growth Hormone?" IRB: Ethics\&Human Research 11.1:6-10. Response to RichardB. Miller's Children, Ethics, and Modern Medicine 161

Tauer, Carol A.

1994 "The NIH Trials of Growth Hormone for Short Stature." IRB: Ethics \& Human Research 16.3: $1-9$.

White, Gladys B.

1993 "Human Growth Hormone: The Dilemma of Expanded Use in Children." Kennedy Institute of Ethics Journal 3.4:4019. 